\title{
BOUNDED GENERATORS IN LINEAR TOPOLOGICAL SPACES
}

\author{
by S. O. IYAHEN* \\ (Received 29 April, 1969; revised 24 November, 1970)
}

1. Ito and Seidman in [5] define a BG space as a locally convex space in which there exists a bounded set with a dense span. In this note we extend the idea to a class of not necessarily locally convex linear topological spaces (1.t.s.). We note the link between the idea of a BG space and Weston's characterization in [7] of separable Banach spaces. Finally we examine $\sigma-B G$ spaces; here the bounded set in the definition of a BG space is replaced by the union of a sequence of bounded sets.

2. Let $A$ be a subset of a linear space $E$. If $k \geqq 2$ and $A+A \subseteq k A$, then $A$ is called a $k$-convex set. An 1.t.s. which has a base of neighbourhoods of the origin consisting of balanced $k$-convex sets (for some fixed $k$ ) is called a $k$-convex 1.t.s.

Every locally convex space is a $k$-convex 1.t.s. for any $k \geqq 2$. Also, a locally bounded space (i.e. an 1.t.s. which has a bounded neighbourhood) is a $k$-convex l.t.s. for some $k \geqq 2$. Thus a $k$-convex l.t.s. need not be a locally convex space. If for some fixed $k, E_{\alpha}$ is a $k$-convex 1.t.s. for each $\alpha$ in an index set $\Psi$, then the product space $X\left(E_{\alpha}: \alpha \in \Psi\right)$ is a $k$-convex 1.t.s. However the product of a sequence of complete Hausdorff locally bounded spaces need not be a $k$-convex l.t.s. for any $k$ (see for example, p. 179 of [6]).

In [6, p. 170] Simons defines the notion of a $\lambda$-pseudometric. It is clear from [6, Theorem 4] that if $(E, u)$ is a $k$-convex l.t.s., then for some $\lambda$, there is a family $\left(p_{\alpha}\right)$ of $u$-continuous $\lambda$-pseudometrics which give the topology $u$.

Let $E$ be a $k$-convex 1.t.s. As in [5], let $\Phi(E)$ denote the set of all families $\varphi=\left(\varphi_{\gamma}\right)$ of continuous $\lambda$-pseudometrics (for some fixed $\lambda$ ) which give the topology of $E$, and if $\varphi=\left(\varphi_{\gamma}\right) \in \Phi(E)$, let

$$
S_{\varphi}=\left(x: x \in E, \sup \varphi_{\gamma}(x)<\infty\right) .
$$

The proof of the equivalence of (A) and (B) in Theorem 1 of [5] goes through for a $k$-convex 1.t.s. if we replace " seminorm" by " $\lambda$-pseudometric" throughout. We use the fact $[6$, Theorem 6] that if $\left(\varphi_{\gamma}\right) \in \Phi(E)$, then a subset $A$ of $E$ is bounded if and only if $\varphi_{\gamma}(A)$ is bounded for each $\gamma$.

If we call an 1.t.s. which contains a bounded set with dense span, a BG space, we immediately have the following generalization of the corollary of Theorem 1 of [5].

- THEOREM 1. With the notation above, a Hausdorff $k$-convex l.t.s. $E$ is a BG space if and only if there is $\varphi$ in $\Phi(E)$ such that $S_{\varphi}$ is dense in $E$.

LEMMA 1. If $A$ is a balanced $k$-convex bounded set in an l.t.s. $(E, u)$, then the family $\left(k^{-n} A: n=1,2, \ldots\right)$ of sets is a base of neighbourhoods for a locally bounded $k$-convex topology $v_{A}$ on the linear span $E_{A}$ of $A$ which is finer than the $u$-induced topology. The space $\left(E_{A}, v_{A}\right)$ is Hausdorff and complete if $(E, u)$ is Hausdorff sequentially complete and $A$ is $u$-closed.

- Present address: Institute of Technology, Benin City, Nigeria. 
For the situation of a locally convex space $(E, u)$, Lemma 1 is well known (see for example the proof of Chapter III, Section 3, No. 4, Lemma 1 of [1]).

It is easy to see that in a $k$-convex 1.t.s., any bounded set is contained in a balanced $k$-convex bounded set.

THEOREM 2. A (sequentially complete) Hausdorff $k$-convex l.t.s. $(E, u)$ is a BG space if and only if there is a one-to-one continuous linear map $t$ from a (complete) Hausdorff locally bounded $k$-convex l.t.s. $F$ into $(E, u)$ such that $t(F)$ is u-dense.

Proof. If $(E, u)$ is a BG space, let $A$ be a balanced $k$-convex bounded set which has a dense span. With $\left(E_{A}, v_{A}\right)$ as in Lemma 1 , let $t$ be the identity map from $\left(E_{A}, v_{A}\right)$ into $(E, u)$.

Given a subset $A$ of an 1.t.s. $E$, and $k \geqq 2$ a fixed real number, the intersection $C$ of the non-empty set of all (closed), balanced $k$-convex subsets of $E$ containing $A$ is (closed) balanced and $k$-convex. The set $C$ is called the (closed) balanced $k$-convex envelope of $A$.

Lemma 2. In a Hausdorff $k$-convex l.t.s. $E$, the balanced $k$-convex envelope $C$ of a precompact set $A$ is precompact.

Proof. Let $U$ be an open balanced $k$-convex neighbourhood in $E$. Since $A$ is precompact, there is a finite subset $B$ of $E$ such that $A \subseteq B+U$, and therefore $C \subseteq B^{\prime}+U$, where $B$ is a compact set, being the closed absolutely convex envelope of the finite set $B$. As $B^{\prime}$ is compact and $U$ is open, there is a finite subset $D$ of $B^{\prime}$ such that $C \subseteq D+U$.

Let us call a linear map from one l.t.s. $G$ into another $H$ a precompact (compact) map if there is a neighbourhood which is mapped into a precompact (compact) set in $H$.

Weston in [7] proves that a Banach space $(E, u)$ is separable if and only if there is a one-to-one compact map $t$ say, from a Banach $F$ into $(E, u)$ such that $t(F)$ is $u$-dense. It is shown in [2] that this result is still valid if " Banach space " is replaced by " complete Hausdorff locally bounded space".

THEOREM 3. A (complete metrizable) metrizable k-convex l.t.s. $(E, u)$ is separable if and only if there is a one-to-one (compact) precompact linear map $t$ say, from a (complete) Hausdorff locally bounded $k$-convex l.t.s. $F$ into $(E, u)$ such that $t(F)$ is $u$-dense.

Proof. Let $(E, u)$ be a separable metrizable $k$-convex 1.t.s. and let $\left(U_{n}\right)$ be a shrinking base of $u$-neighbourhoods. If $\left(x_{n}: n=1,2, \ldots\right)$ is a countable $u$-dense subset of $E$, then for each $n$, there is a non-zero real number $\alpha_{n}$ such that $\alpha_{n} x_{n} \in U_{n}$. As $\left(U_{n}\right)$ is shrinking, the sequence $\left(\alpha_{n} x_{n}\right)$ thus converges to zero in $(E, u)$. By Lemma 2, the balanced $k$-convex envelope $A$ of $\left(\alpha_{n} x_{n}: n=1,2, \ldots\right)$ is precompact; its closure is compact if $(E, u)$ is complete. We now apply Lemma 1 . With $F=\left(E_{A}, v_{A}\right)$, the identity map $t$ from $F$ into $(E, u)$ is precompact, being compact if $(E, u)$ is complete.

COROLlary. A separable infinite dimensional Fréchet space contains a dense subspace on which there is a finer Fréchet space topology.

THEOREM 4. A complete metrizable $k$-convex l.t.s. $(E, u)$ is finite dimensional if and only if $t(F)$ is closed in $(E, u)$ whenever $t$ is a continuous linear map from a complete metrizable $k$-convex l.t.s. $F$ into $(E, u)$. 
Proof. Suppose first that $(E, u)$ is separable. Then by Theorem 3, there is a one-to-one compact (and therefore continuous) linear map $t$ say, from a complete metrizable $k$-convex l.t.s. $F$ into $(E, u)$ such that $t(F)$ is $u$-dense. If $t$ has a closed range, $t(F)=E$ and $t$ is a topological isomorphism by Banach's inversion theorem. Therefore $(E, u)$ has a compact neighbourhood and is thus finite dimensional.

If $(E, u)$ is not necessarily separable, let $E_{0}$ be a subspace of $E$ of countable dimension. Let $E_{1}$ be the closure of $E_{0}$ in $E$ and let $u_{1}$ be the $u$-induced topology on $E_{1}$. Then $\left(E_{1}, u_{1}\right)$ is a separable complete metrizable $k$-convex l.t.s. If $t(F)$ is closed in $(E, u)$ whenever $t$ is a continuous linear map from a complete metrizable $k$-convex 1.t.s. $F$ into $(E, u)$, then by the argument above, the dimension of $E_{1}$ is necessarily finite. The dimension of $E$ must then be finite, otherwise, we could choose $E_{0}$ as above to have countably infinite dimension.

COROLlaRY. If $E$ is a Fréchet space and every continuous linear map from any Fréchet space into $E$ has a closed range then $E$ is finite dimensional.

Ito and Seidman in [5, p. 287] call a Hausdorff locally convex space $E$ a HBG space if every closed linear subspace of $E$ is a $\mathrm{BG}$ space. Let $(E, u)$ be a normed linear space of infinite dimension. If $v$ is the weak topology associated with $u$, then it follows from [5, Theorem $2(D)]$ that $(E, v)$ is a $\mathrm{BG}$ space. In fact $(E, v)$ is a $\mathrm{HBG}$ space. As $(E, v)$ is not quasibarrelled, $(E, v)$ is not the quotient of a product of normed linear spaces.

Cf. [5, p. 287, questions 2 and 3].

3. Let $E$ be an 1.t.s. We call $E$ a $\sigma-\mathrm{BG}$ space if there is a sequence of bounded sets, the union of which spans a dense subspace of $E$.

Every BG space is a $\sigma$-BG space. Also, every separable 1.t.s. is a $\sigma$-BG space.

If $E$ is a linear space of countably infinite dimension, then under its finest locally convex topology $\tau\left(E, E^{*}\right), E$ is separable (and complete) and therefore the space $\left(E, \tau\left(E, E^{*}\right)\right)$ is a $\sigma$-BG space. As each $\tau\left(E, E^{*}\right)$-bounded set is contained in some finite dimensional linear subspace of $E,\left(E, \tau\left(E, E^{*}\right)\right)$ is not a $\mathrm{BG}$ space.

It follows from [1, Ch. III, section 2, exercise 5] that a metrizable $k$-convex 1.t.s. is a BG space if and only if it is a $\sigma-B G$ space. The example of Ito and Seidman [5, p. 286] then shows that a Fréchet space need not be a $\sigma-\mathrm{BG}$ space. However as in Theorem 2 of [5], a product of BG $(\sigma-\mathrm{BG})$ spaces is a BG $(\sigma-\mathrm{BG})$ space, and the image under a continuous linear map of a $\mathrm{BG}(\sigma \mathrm{BG})$ space is of the same sort.

For a fixed $k \geqq 2$ and each positive integer $n$, let $\left(E_{n}, u_{n}\right)$ be a $k$-convex I.t.s. such that $E_{n} \subset E_{n+1}$. If $E=\bigcup_{n}\left(E_{n}\right)$, then there is a finest linear topology $u$ say, on $E$ such that each identity map $\left(E_{n}, u_{n}\right) \rightarrow E$ is continuous [4, Definition 2.1]. By an application of Proposition 2.2 of [4], we see that $(E, u)$ is a $k$-convex 1.t.s., and that if each $\left(E_{n}, u_{n}\right)$ is locally convex, so is $(E, u)$. The space $(E, u)$ is called the generalized strict $k$-convex inductive limit of $\left(E_{n}, u_{n}\right)$. If in addition, each $u_{n}$ coincides with the topology induced on $E_{n}$ by $u_{n+1}$, then $(E, u)$ is called the strict $k$-convex inductive limit of $\left(E_{n}, u_{n}\right)$.

If $(E, u)$ is the strict $k$-convex inductive limit of $\left(E_{n}, u_{n}\right)$, then the topology $u$ coincides with $u_{n}$ on each $E_{n},(E, u)$ is Hausdorff if each $\left(E_{n}, u_{n}\right)$ is [4, Proposition 2.7, Cor. 1], and in this case if each $\left(E_{n}, u_{n}\right)$ is complete, $(E, u)$ is also complete [4, Proposition 2.8, Cor.], but is not metrizable [4, Proposition 2.9, Cor.]. We shall prove: 
THEOREM 5. The strict $k$-convex inductive limit of a sequence of complete Hausdorff $k$-convex $\sigma$-BG spaces is also a $\sigma$-BG space.

This theorem will follow immediately from the following result.

Lemma 3. If $(E, u)$ is the strict $k$-convex inductive limit of $\left(E_{n}, u_{n}\right)$ where each $\left(E_{n}, u_{n}\right)$ is complete, $k$-convex and Hausdorf, then a subset of $E$ is $u$-bounded if and only if it is contained in some $E_{n}$ and is $u_{n}$-bounded.

Proof. Suppose that $A$ is a $u$-closed balanced $k$-convex $u$-bounded set which is not contained in any $E_{n}$. Then there is a subsequence $(n(i))$ of $(n)$ such that for each $i$, some point of $A \cap E_{n(i+1)}$ is not in $E_{n(i)}$ and $(E, u)$ is the strict $k$-convex inductive limit of $\left(E_{n(i)}, u_{n(i)}\right)$. Observe that $(E, u)$ is complete and Hausdorff and that each $E_{n(i)}$ is $u$-closed.

As in Lemma 1, let $E_{A}$ be the linear span of $A$ and $v_{A}$ the linear topology on $E_{A}$ with the family $\left(k^{-m} A: m=1,2, \ldots\right)$ of sets as a base of neighbourhoods. Similarly, let $F_{n(i)}$ be the linear span of $A \cap E_{n(i)}$ and $v_{n(i)}$ the linear topology on $F_{n(i)}$ with the family $\left(k^{-m}\left(A \cap E_{n(i)}\right)\right.$ : $m=1,2, \ldots)$ of sets as a base of neighbourhoods. The spaces $\left(E_{A}, v_{A}\right),\left(F_{n(i)}, v_{n(i)}\right)$ are complete Hausdorff locally bounded $k$-convex spaces, $F_{n(1)} \subset F_{n(2)} \subset F_{n(3)} \subset \ldots, E_{A}=\bigcup F_{n(i)}$, and $v_{n(i)}$ coincides with the $v_{n(i+1)}$-induced topology on $F_{n(i)}$. If $\left(E_{A}, w\right)$ is the strict $k$-convex inductive limit of $\left(F_{n(i)}, v_{n(i)}\right), w$ is finer than the $u$-induced topology on $E_{A}$, and it follows that the identity map from $\left(E_{A}, w\right)$ onto $\left(E_{A}, v_{A}\right)$ has a closed graph. By Theorem 4.2 of [3], we see that $v_{A}=w$, implying that $\left(E_{A}, w\right)$ is metrizable. As this is not possible, the set $A$ must be contained in some $E_{n}$, and is $u_{n}$-bounded because $A$ is $u$-bounded and $u$ induces the topology $u_{n}$ on each $E_{n}$.

Thus any strict inductive limit of a sequence of Banach or separable Fréchet spaces is a $\sigma$-BG space. Also, if $E$ is the sequence space $l^{p}(0<p<1)$ and $F$ is the algebraic direct sum of countably many copies of $E$, then under the finest linear topology for which the injection maps $E \rightarrow F$ are continuous, $F$ is a $\sigma$-BG space.

There is a parallel to Theorem 2 .

THEOREM 6. If a (sequentially complete) Hausdorff $k$-convex l.t.s. $(E, u)$ is a $\sigma-\mathrm{BG}$ space but not a BG space, then there is a one-to-one continuous linear map, $t$ say, from $F$ into $(E, u)$ such that $t(F)$ is $u$-dense, where $F$ is the generalized strict $k$-convex inductive limit of a sequence of (complete) Hausdorff locally bounded spaces.

Proof. Let a Hausdorff $k$-convex 1.t.s. $(E, u)$ be a $\sigma$-BG space but not a BG space. Let $\left(A_{n}\right)$ be a sequence of $u$-closed balanced $k$-convex $u$-bounded sets, the union of which spans a dense linear subspace $F$ of $(E, u)$. We may assume that $A_{1} \subset A_{2} \subset A_{3} \subset \ldots$; and since no $A_{n}$ spans $F$, we may further assume that for each $n, A_{n+1} \$ E_{A_{n}}$. If $v_{A_{n}}$ is the topology on $E_{A_{n}}$ defined as in Lemma 1, let $(F, v)$ be the generalized strict $k$-convex inductive limit of $\left(E_{A_{n}}, v_{A_{n}}\right)$ and let the map $t: F \rightarrow F$ be the identity map.

The author wishes to thank the referee for his suggestions.

\section{REFERENCES}

1. N. Bourbaki, Éléments de mathématique, Livre V; Espaces vectoriels topologiques, Ch. III-V, Actualités Sci. Ind. 1229 (Paris, 1955). 

95-96.

2. S. O. Iyahen, A note on separable locally bounded spaces, Nigerian J. of Science 3 (1969),

3. S. O. Iyahen, $D(\tau ; l)$-spaces and the closed graph theorem, Proc. Edinburgh Math. Soc. (2) 16 (1968), 89-99.

4. S. O. Iyahen, On certain classes of linear topological spaces, Proc. London Math. Soc. (3) 18 (1968), 285-307.

5. T. Ito and T. Seidman, Bounded generators of linear spaces, Pacific J. Math. 26 (1968), 283-287.

6. S. Simons, Boundedness in linear topological spaces, Trans. Amer. Math. Soc. 113 (1964), 169-180.

7. J. D. Weston, A characterization of separable Banach spaces, J. London Math. Soc. 32 (1957), 186-187.

UNIVERSITY OF IBADAN

NIGERIA 